\title{
Effects of chronic folate deficiency and sex differences on depression-like behavior in mice
}

\author{
WANXIAO SUN*, QITING QING* , XU CHENG, JING CHEN, NINGNING YU, LIYUAN ZHU and MEI ZHAO \\ Department of Basic Nursing, School of Nursing, Anhui Medical University, Hefei, Anhui 230032, P.R. China
}

Received September 11, 2021; Accepted December 3, 2021

DOI: $10.3892 /$ etm.2022.11129

\begin{abstract}
Although previous studies have reported that serum folate levels are negatively associated with depression in women but not men, it remains unclear whether folate deficiency can directly lead to depression and whether sex difference serves a role in this condition, since the potential mechanism remains elusive. Therefore, the present study aimed to investigate whether folate deficiency results in differences in parameters associated with depression between males and females. CD-1 mice received either a standard control diet or a folate-deficient diet from 10 to 38 weeks of age, following which behavioral assays, such as an open field test, sucrose preference test and forced swim test were performed throughout week 38 . Serum and cerebral cortex samples were subsequently collected for assessment. Serum folate, homocysteine, estradiol (E2) and testosterone levels were measured using chemiluminescence, enzymatic cycling assay and electrochemiluminescence immunoassays. The cerebral cortex was used for western blot analysis, to detect the expression levels of estrogen receptor $\beta(\mathrm{ER} \beta), \mathrm{PI} 3 \mathrm{~K} / \mathrm{AKT}$ pathway and caspase-3. The results revealed that compared with those in female mice that received standard control diet, female mice that received folate-deficient diet exhibited lower E2 concentrations, lower sucrose preferences (as determined through the sucrose preference test), longer durations of immobility (as determined in the forced swim test) and less time spent in the central areas of the open field test. Western blotting demonstrated that the expression levels of ER $\beta$ and the phosphorylation levels of PI3K and AKT were decreased, whilst the expression levels of cleaved caspase- 3 were increased, in the cerebral cortex of female mice that received folate-deficient diet. However, no differences in E2 concentration, behavioral assay parameters
\end{abstract}

Correspondence to: Professor Mei Zhao, Department of Basic Nursing, School of Nursing, Anhui Medical University, 81 Meishan Road, Hefei, Anhui 230032, P.R. China

E-mail: zhaomei@ahmu.edu.cn

${ }^{*}$ Contributed equally

Key words: depression, folate, sex differences, estradiol, caspase-3, neuronal apoptosis or protein levels of ER $\beta$, phosphorylated (p-)PI3K, p-AKT and cleaved caspase- 3 could be observed in male mice regardless of whether they received standard control or folate-deficient diets. Collectively, these results revealed that folate deficiency only led to depression-like behavior in female mice. This may be associated with reduced E2 levels, which may inhibit the PI3K/AKT pathway and upregulate the expression of cleaved caspase-3 to promote neuronal apoptosis.

\section{Introduction}

Depression is a prevalent mental condition that is characterized by depressed mood, anhedonia, fatigue or loss of energy, weight loss, psychomotor retardation or agitation $(1,2)$. It affects $15-18 \%$ of the population and is generally more prevalent in women (3-5). Depression is a leading cause of reduction in the quality of life. Furthermore, the World Health Organization projected that depression would rank as the leading contributor to the global burden of disease by $2030(1,2)$.

Emerging evidence has revealed that folate deficiency is a risk factor for depression $(6,7)$. Folate refers to a group of water-soluble compounds that are also known as vitamin B9 (8). It is considered to be associated with the function and development of the central nervous system (8). Previous studies have suggested that individuals with depression exhibited lower serum folate levels compared with those in individuals without depression (7,9,10). Additionally, a sex-stratified analysis of the National Health and Nutrition Examination Survey data revealed that serum folate levels are negatively associated with depression in women but not in men (11). However, to the best of our knowledge, no studies have investigated the effects of folate deficiency on depression-like behavior in the two sexes. Therefore, it is necessary to determine the relationship between chronic folate deficiency and depression, in addition to establishing if there are sex-associated differences related to this condition.

The mechanism by which folate deficiency affects female-associated depression has yet to be fully determined. However, a previous study reported that folate deficiency can lead to reduced estradiol (E2) levels (12). It has also been documented that women are more susceptible to depression when estrogen concentrations are low $(13,14)$. Previous studies have demonstrated that plasma E2 levels tended to be significantly lower among women with depression, suggesting that E2 supplementation can be beneficial for the treatment of 
depression (15-17). Another study suggested that E2 serves a role in depression (13), where the effects mediated by E2 on depressive behavior may involve estrogen receptor $\beta(\mathrm{ER} \beta)$ signaling. Apart from estrogen receptor $\alpha(E R \alpha)$, which serves an important role in neuroendocrine reproductive function (18), $\mathrm{ER} \beta$ is more likely to affect $\operatorname{mood}(18) . \mathrm{ER} \beta$ is a nuclear receptor that is localized to the cell membrane and is widely distributed in the cerebral cortex whilst also being present in both neurons and glia (18-20). E2 can cross the blood-brain barrier and exert its actions through ER $\beta$ to regulate the PI3K/AKT signaling pathway (18). PI3K/AKT signaling is ubiquitous in cells and regulates the proliferation and differentiation of cells (21-23). Its downregulation or inhibition can increase cleaved caspase-3 expression, which promotes neuronal apoptosis $(24,25)$. In individuals with depression, it has been shown that neuronal cell density is reduced in the cerebral cortex (26).

By applying animal models, the present study aimed to investigate whether folate deficiency can result in differences in parameters associated with depression between males and females. In addition, another aim of the present study was to elucidate the potential underlying mechanism by which this occurs. It was hypothesized that a chronic folate-deficient diet may reduce $\mathrm{E} 2$ levels in mice, leading to neuronal apoptosis in the cerebral cortex to induce depression-like behavior, by inhibiting the expression of ER $\beta$ and suppressing the PI3K/AKT signaling pathway.

\section{Materials and methods}

Folic acid diet groups. Folic acid is a derivative of folates due to the high chemical lability of naturally occurring folates, which is used in supplements and for food fortification $(8,27)$. Based on the standard AIN-93G diet (28), two folic acid diet (cat. nos. LAD-3001G-F2 and LAD-3001G-F0; Trophic Animal Feed High-Tech Co., Ltd.) groups were designed: i) The control diet (consisting of $2 \mathrm{mg} / \mathrm{kg}$ folic acid); and ii) the chronic folate-deficient (CFD) diet, consisting of $0 \mathrm{mg} / \mathrm{kg}$ folic acid. It is generally accepted that $2 \mathrm{mg} / \mathrm{kg}$ folic acid is the basic requirement for rodents (28). In addition to the difference in folic acid content between the two diets, the CFD diet contained succinyl sulfathiazole (1\%), which inhibits folic acid production by gut bacteria (29), ensuring folic acid deficiency in mice in the CFD group.

Animals and treatments. A total of 40 male (weight, 31-33 g) and 40 female (weight, 30-32 g) CD-1 mice were purchased from Beijing Vital River Laboratory Animal Technology Co., Ltd. at 8 weeks of age. All procedures performed on animals were in accordance with the guidelines for humane treatment set by the Association of Laboratory Animal Sciences and the Center for Laboratory Animal Science at Anhui Medical University (GB/T 35892-2018) (30). The present study was approved by the Ethics Committee of Anhui Medical University (approval no. LLSC20150350; Hefei, China). A total of four mice were housed per cage under a 12-h dark/light cycle in a controlled environment (temperature, $20-24^{\circ} \mathrm{C}$; relative humidity, $50-55 \%$ ) and left to acclimatize for 2 weeks. Standard control diet and water were available ad libitum during this time. Bahous et al (31) previously indicated that a folate-deficient diet administered to mice for $\sim 30$ weeks reduced the duration spent in the center areas of an open field test due to long-term folate deficiency (31). Therefore, mice in the present study were administered the control or folate-deficient diet from 10 to 38 weeks of age (totaling 28 weeks).

Mice at 10 weeks of age were randomly divided into four groups (n=20 per group) as follows: i) Female control group (F-Ctrl); ii) female chronic folate deficiency group (F-CFD); iii) male control group (M-Ctrl); and iv) male chronic folate deficiency group (M-CFD). F-Ctrl and M-Ctrl mice were fed with a controlled diet, whilst F-CFD and M-CFD mice received a folate-deficient diet. The body weight of each mouse was recorded every 7 days. Behavioral assessments were performed on mice during their 38th week. All mice were subsequently sacrificed by dislocation of cervical vertebra on the second day following the behavioral experiments. After sacrifice, serum samples of all mice were collected, centrifuged at $12,000 \mathrm{x} \mathrm{g}$ for $15 \mathrm{~min}$ at $4^{\circ} \mathrm{C}$ and stored at $-80^{\circ} \mathrm{C}$. Serum folate, homocysteine, E2 and testosterone levels were then measured. In the present study, considering that stimulation may cause changes in the mouse brain, such as decreased protein expression in the medial prefrontal cortex (32), cerebral cortex samples of mice that were not subjected to behavioral assays were extracted following sacrifice and were stored at $-80^{\circ} \mathrm{C}$ for subsequent experimentation.

Behavioral assays. Mice that underwent the aforementioned diet regimens were used for behavioral testing from during the 38th week of age ( $n=10$ per group). The experiments were performed in the following sequence: i) Open field test (OFT); ii) sucrose preference test (SPT); and iii) forced swim test (FST). All behavioral tests were performed during the light cycle of housing in a dedicated sound-proof behavioral facility located in Laboratory Animal Center of Anhui Medical University. All mice were brought to the testing room and underwent $30 \mathrm{~min}$ of acclimatization before the start of each behavioral test and remained in the same room once the test was initiated. Behaviors in OFT and FST were evaluated using SMART software (SMART v3.0.02; Panlab, Inc.). All animals were sacrificed on the second day following FST, to obtain the aforementioned samples.

OFT. The OFT is used to measure the activity of mice in an enclosed open area. Mice that travelled the least distance, spend the shortest amount of time in the center and enter into the center area the fewest times are considered to be more depressed (33). In the present study, mice were gently placed into the same corner of the open field, which consisted of a wooden box $(46 \times 46 \times 40 \mathrm{~cm})$ with its floor marked into 25 squares (Fig. S1). Of these, nine squares were defined as the center whereas the outer 16 squares along the walls were defined as the periphery. A 5-min video was then recorded to observe the locomotor activity of mice. The following parameters were assessed during the test: i) Total distance; ii) number of squares crossed; and iii) time spent in each square. Between tests, $75 \%$ alcohol was used to disinfect the chamber.

SPT. The SPT was used to evaluate anhedonia in mice, a core symptom of depression (34). Decreased sucrose preference is considered to indicate depression-like behavior (34). Mice were housed separately and were habituated to two bottles containing 
either tap water or $1 \%$ sucrose solution for 2 days. The location of the two bottles was changed every $12 \mathrm{~h}$ to prevent the possible effects of side preference on drinking behavior. On the day 3 , mice were deprived of water and food for $24 \mathrm{~h}$. The next day, each mouse was given a free choice between two bottles, one with tap water and the other with $1 \%$ sucrose solution. The left/right position of the bottles was alternated after $12 \mathrm{~h}$. Sucrose preference (\%) was calculated using the following formula: Sucrose preference rate $(\%)=$ Sucrose intake $(\mathrm{g}) /[$ Water intake $(\mathrm{g})+$ sucrose intake $(\mathrm{g})] \mathrm{x} 100$.

FST. The FST is used to assess despair in animals. Longer immobility times indicate that a mouse is exhibiting depression (35). The Mice were placed into a vertical transparent cylinder containing temperature-controlled water $\left(24 \pm 1^{\circ} \mathrm{C}\right)$. The cylinder was $20 \mathrm{~cm}$ deep, with a height of $30 \mathrm{~cm}$ and a diameter of $12 \mathrm{~cm}$, to ensure that mice could neither escape nor touch the bottom of the container. The mice were forced to swim for a total of $6 \mathrm{~min}$, during which behavior was monitored over the last $4 \mathrm{~min}$. Immobility time was recorded and analyzed using the aforementioned software, SMART.

Serum detection. Serum samples of all mice were collected through centrifugation at $12,000 \mathrm{x}$ g for $15 \mathrm{~min}$ at $4^{\circ} \mathrm{C}$ and were stored at $-80^{\circ} \mathrm{C}$ once behavioral experiments were completed at the end of 38 weeks of age. Serum concentrations of folate were measured using chemiluminescence (cat. no. A98032; Beckman Coulter, Inc.) according to the manufacturer's instructions (36). Serum concentrations of total homocysteine (HCY) were measured using an enzymatic cycling assay [cat. no. 1.02.4802; Shanghai Fosun Pharmaceutical (Group) Co., Ltd.] (37). Serum E2 (Elecsys Estradiol III; cat. no. 6656021190; Roche Diagnostics GmbH) and testosterone (Elecsys Testosterone II; cat. no. 5200067190; Roche Diagnostics $\mathrm{GmbH}$ ) were measured using electrochemiluminescence immunoassays according to the manufacturer's instructions $(38,39)$.

Western blot analysis. At the end of the behavioral experiments, the cerebral cortex of mice that were not subjected to behavior assays was collected and homogenized using $0.4 \mathrm{ml}$ lysis buffer containing $50 \mathrm{mM}$ Tris- $\mathrm{HCl}, 150 \mathrm{mM} \mathrm{NaCl}$, $1 \mathrm{mM}$ EDTA, $1 \%$ Triton X-100, $1 \%$ sodium deoxycholate and $0.1 \%$ SDS, supplemented with $1 \%$ protease inhibitors, $1 \%$ PMSF and $2 \%$ phosphatase inhibitor. After centrifugation at $15,000 \times \mathrm{g}$ for $15 \mathrm{~min}$ at $4^{\circ} \mathrm{C}$, protein concentrations were measured using BCA assay. The samples were then boiled for $10 \mathrm{~min}$ at $100^{\circ} \mathrm{C}$, after which equal amounts of protein $(30 \mu \mathrm{g})$ were separated by SDS-PAGE (12.5 or $15 \%)$ and transferred onto PVDF membranes. After blocking in blocking buffer (5\% skimmed milk powder) at room temperature for $1.5 \mathrm{~h}$, blots were incubated overnight with antibodies against ER $\beta$ (1:1,000; cat. no. 8974; Santa Cruz Biotechnology, Inc.), phosphorylated (p)-PI3K (1:1,000; cat. no. 4228T; Cell Signaling Technology, Inc.), PI3K (1:1,000; cat. no. 4257S; Cell Signaling Technology, Inc.), p-AKT (1:1,000; cat. no. 4060S; Cell Signaling Technology, Inc.), AKT (1:1,000; cat. no. 4691S; Cell Signaling Technology, Inc.), caspase-3 (1:500; cat. no. 14220T; Cell Signaling Technology, Inc.) and GAPDH (1:1,000; cat. no. 365062; Sant Cruz, Inc.) at $4^{\circ} \mathrm{C}$. After washing three

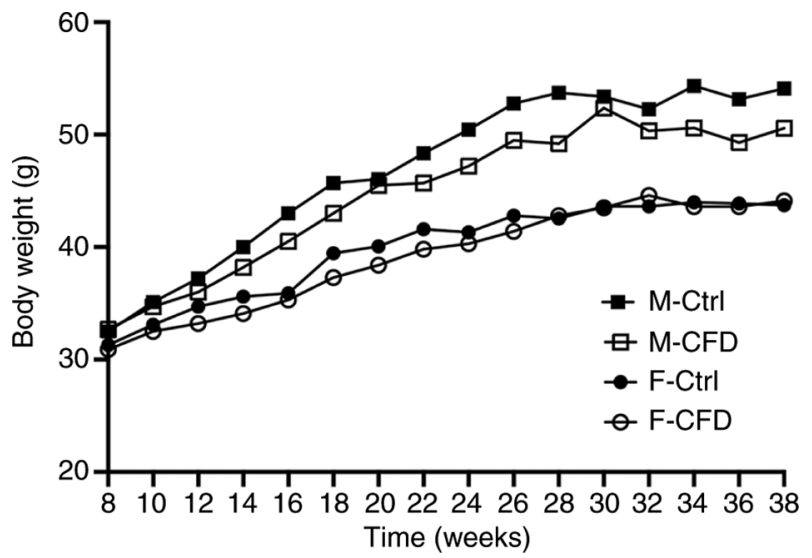

Figure 1. Mouse body weight ( $\mathrm{n}=20$ per group). CFD, chronic folatedeficient; F-Ctrl, female mice with standard control diet; F-CFD, female mice with folate deficiency diet; M-Ctrl, male mice with standard control diet; M-CFD, male mice with folate deficiency diet.

times, the membrane was probed with HRP-conjugated goat anti-rabbit IgG antibodies (1:10,000; cat. no. sc-2005; Santa Cruz Biotechnology, Inc.) for $1 \mathrm{~h}$ at room temperature. The blots were subsequently detected using an ECL detection kit (Pierce; Thermo Fisher Scientific, Inc.) and quantified using a ChemiDoc Imaging system (version, 2.3.0.07; cat. no. 12003154; BioRad, Inc.).

Statistical analysis. SPSS 24.0 (IBM Corp.) and GraphPad Prism 9 (GraphPad Software, Inc.) software were used to analyze data, which were presented as the mean \pm SD. Body weight was analyzed using mixed ANOVA followed by Tukey's test to study the effects of different diets on the weight of mice over time. The remaining data were analyzed using an unpaired two-tailed Student's t-test. $\mathrm{P}<0.05$ was considered to indicate a statistically significant difference.

\section{Results}

CFD diet does not change murine body weight but increases homocysteine concentrations. Body weight was recorded every week. Fig. S2 is the result of statistical analysis, which represents the influence of age factors (intra-group factors) on the body weight of mice, and its weight value is the same as Fig. 1. Mixed ANOVA analysis revealed that the body weights of mice in the F-Ctrl $(\mathrm{P}<0.01)$, F-CFD $(\mathrm{P}<0.01)$, M-Ctrl $(\mathrm{P}<0.01)$ and $\mathrm{M}-\mathrm{CFD}(\mathrm{P}<0.01)$ groups increased with age (Fig. S2). However, the factor of diet did not induce a statistically significant difference in the weight of female $(\mathrm{P}=0.70)$ or male mice $(\mathrm{P}=0.16$; Fig. 1$)$. In conclusion, there was no statistical difference in body weight in both female and male mice on a folate-deficient diet.

Serum folate and homocysteine were measured to investigate the effect of chronic folate deficiency. Folate is a water-soluble vitamin that serves a fundamental role as a methyl donor in the re-methylation of homocysteine to produce methionine (40). During folate deficiency, increased levels of homocysteine are observed in humans (40). Therefore, serum homocysteine can be used as a useful functional indicator of the folate status (40). In the present study, after animals 
A

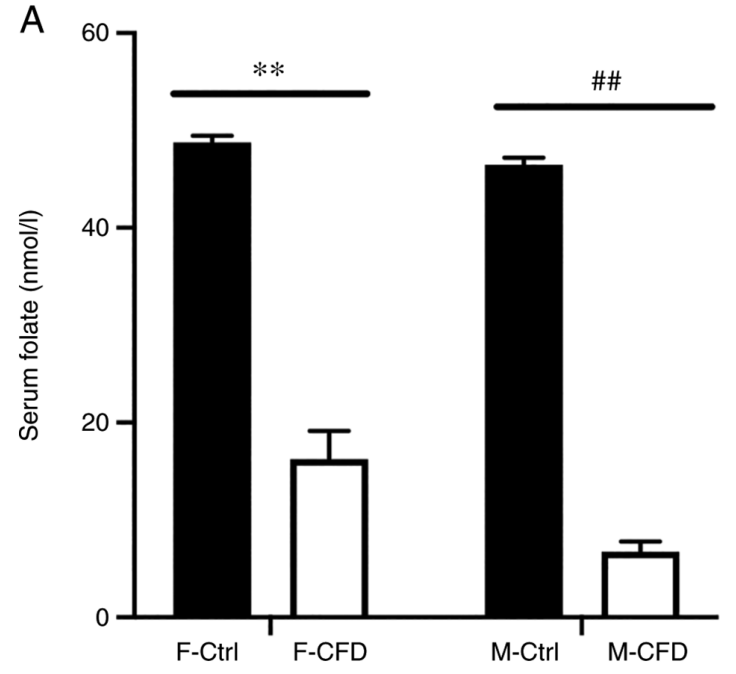

B

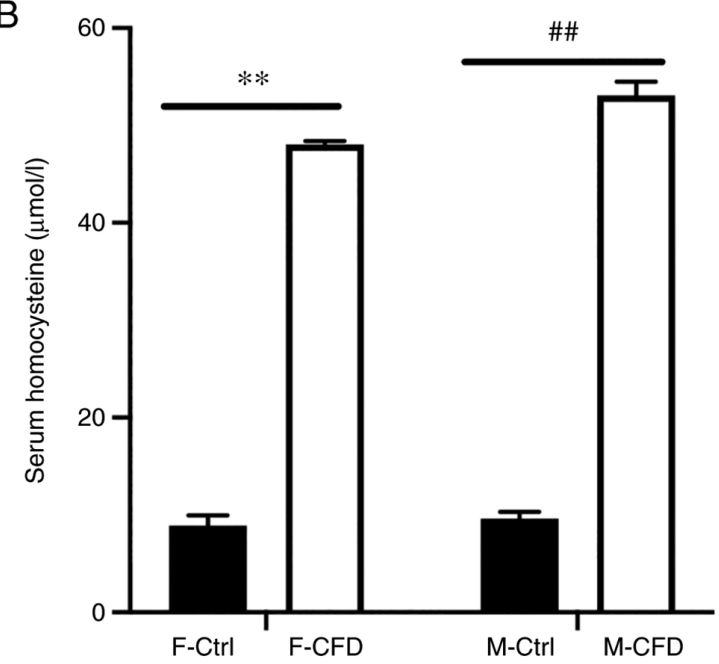

Figure 2. CFD diet increases homocysteine concentrations in mice. All mice were fed with either the folate-deficient diet or standard control diet from 10 to 38 weeks of age, before serum folate and homocysteine concentrations were detected at the end of week 38. (A) Serum folate concentrations in mice ( $\mathrm{n}=20$ per group). (B) Serum total homocysteine concentrations in mice ( $n=20$ per group). ${ }^{* *} \mathrm{P}<0.01$ and ${ }^{\# \#} \mathrm{P}<0.01$. Data are presented as the mean \pm SD. $C F D$, chronic folate-deficient; F-Ctrl, female mice with standard control diet; F-CFD, female mice with folate deficiency diet; M-Ctrl, male mice with standard control diet; M-CFD, male mice with folate deficiency diet.
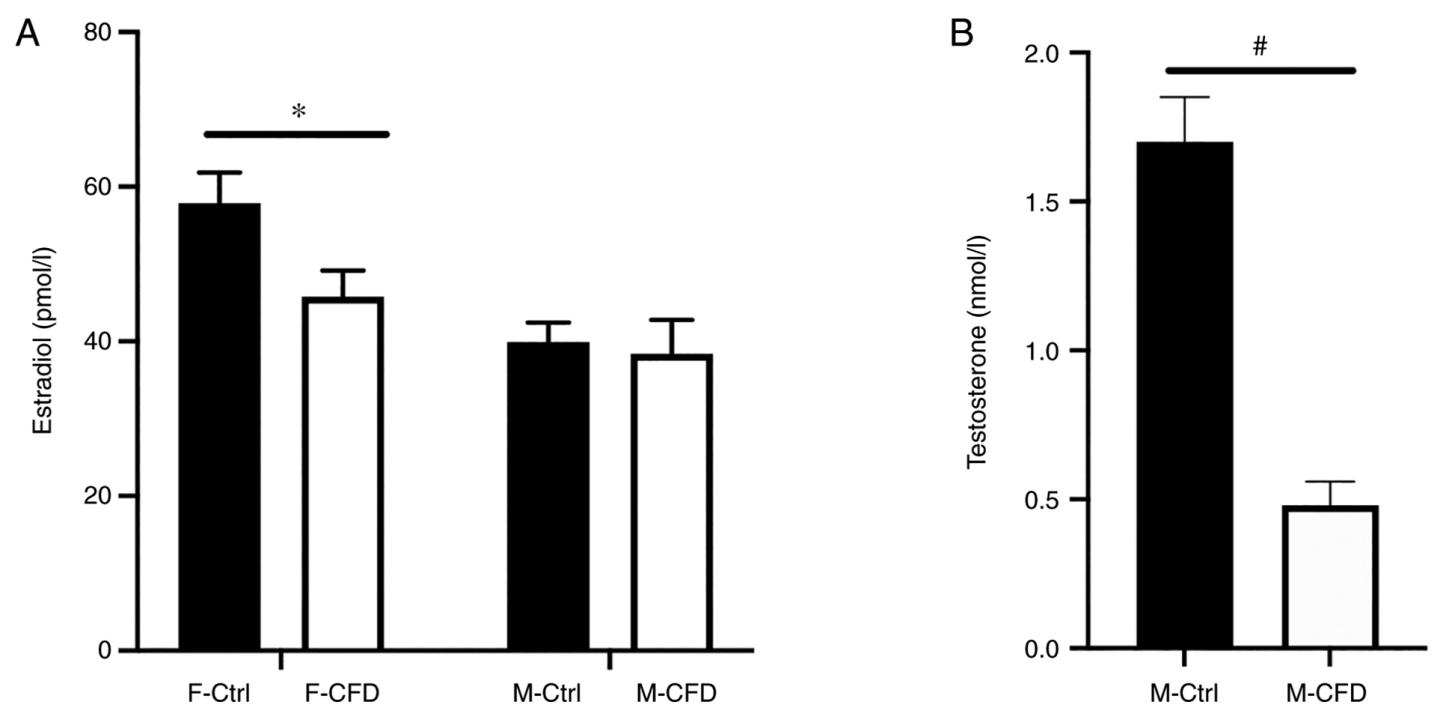

Figure 3. CFD diet decreases estradiol levels in female mice. All mice were fed with either the folate-deficient diet or standard control diet from 10 to 38 weeks of age, before serum estradiol and testosterone concentrations were detected after behavioral assays at the end of week 38. (A) Estradiol concentrations in mice ( $\mathrm{n}=20$ per group). (B) Testosterone concentrations in male mice ( $\mathrm{n}=20$ per group). ${ }^{*} \mathrm{P}<0.05$ and ${ }^{\#} \mathrm{P}<0.05$. Data are presented as the mean $\pm \mathrm{SD}$. CFD, chronic folate-deficient; F-Ctrl, female mice with standard control diet; F-CFD, female mice with folate deficiency diet; M-Ctrl, male mice with standard control diet; M-CFD, male mice with folate deficiency diet.

received a folate-deficient diet, both F-CFD $(\mathrm{P}<0.01)$ and M-CFD ( $\mathrm{P}<0.01)$ mice exhibited significantly lower serum folate concentrations compared with those in their respective controls (Fig. 2A). Accordingly, serum homocysteine levels were significantly higher in mice in the F-CFD $(\mathrm{P}<0.01)$ and $\mathrm{M}-\mathrm{CFD}(\mathrm{P}<0.01)$ groups compared with those in their respective controls (Fig. 2B). In summary, a folate-deficient diet caused reduced concentrations of serum folate and increased concentrations of homocysteine in F-CFD and M-CFD mice.

CFD diet reduces E2 concentrations in female mice. Serum E2 levels were measured in the mice of the present study. Following administration of the CFD diet, E2 levels were significantly reduced in female mice $(\mathrm{P}<0.05$; Fig. $3 \mathrm{~A})$. However, the CFD diet did not affect the E2 levels $(\mathrm{P}=0.760)$ of male mice, even though testosterone concentrations were significantly lower in the M-CFD group compared with those in the $\mathrm{M}$-Ctrl group $(\mathrm{P}<0.05$; Fig. $3 \mathrm{~A}$ and $\mathrm{B})$. In conclusion, folate-deficient diet caused lower E2 levels only in F-CFD.

CFD diet leads to depression-like behaviors in female mice. To assess whether folate deficiency could lead to depression-like behavior, OFT, SPT and FST were performed to evaluate the extent of depression-associated behaviors in mice following the administration of the two different diets. OFT results revealed that F-CFD mice spent significantly less time in 

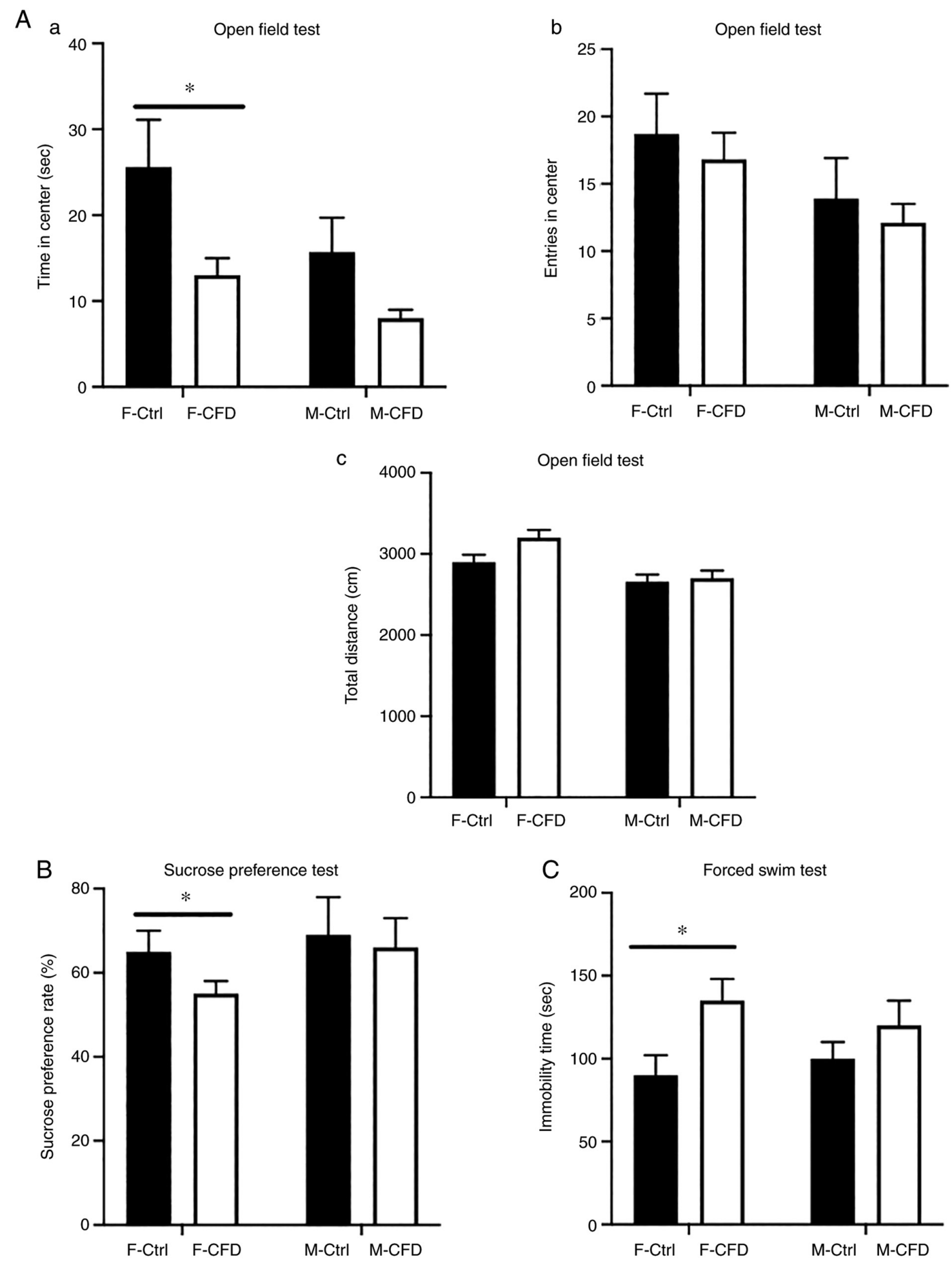

Figure 4. CFD diet leads to depression-like behavior in female mice. All mice were fed with either the folate-deficient diet or standard control diet from 10 to 38 weeks of age, before all behavioral tests were performed from 38 weeks to the end of 38 weeks of age ( $\mathrm{n}=10$ per group). (Aa) Time spent in the center areas of the OFT. (Ab) Number of entries into the center areas in the OFT. (Ac) Total distance travelled in the OFT. (B) Sucrose preference rate of mice in the SPT. (C) Mean time of immobility in the FST. "P<0.05. Data are presented as the mean \pm SD. OFT, open field test; SPT, sucrose preference test; FST, forced swim test; CFD, chronic folate-deficient; F-Ctrl, female mice with standard control diet; F-CFD, female mice with folate deficiency diet; M-Ctrl, male mice with standard control diet; M-CFD, male mice with folate deficiency diet.

the central areas compared with time spent by F-Ctrl mice (Fig. 4Aa), suggesting that the CFD diet caused a decrease in exploratory behavior. No statistically significant differences in the total distance traveled or entries into the center could be observed in either F-CFD or M-CFD mice when compared with their respective controls (Fig. 4Ab and Ac). In addition, the CFD diet resulted in significantly reduced sucrose preference to $<60 \%$ in female mice, an effect not observed in males (Fig. 4B). FST revealed that administration of the CFD diet significantly increased the immobility time of female mice 

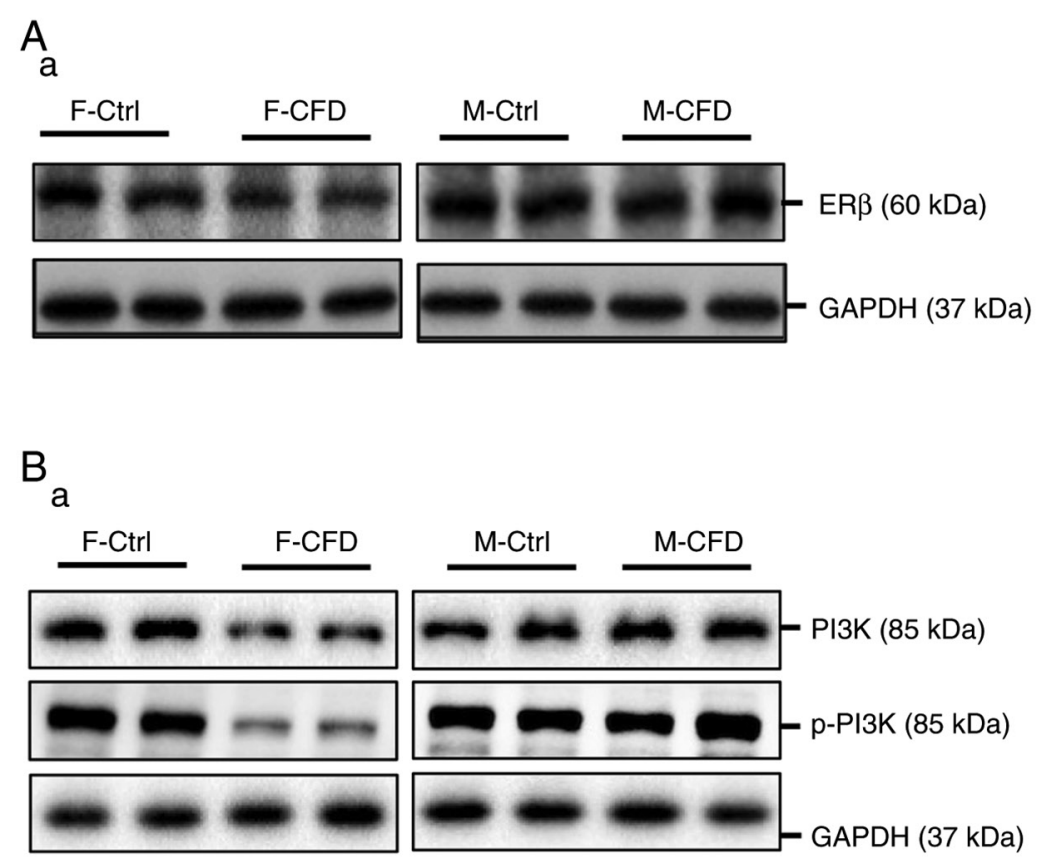

C

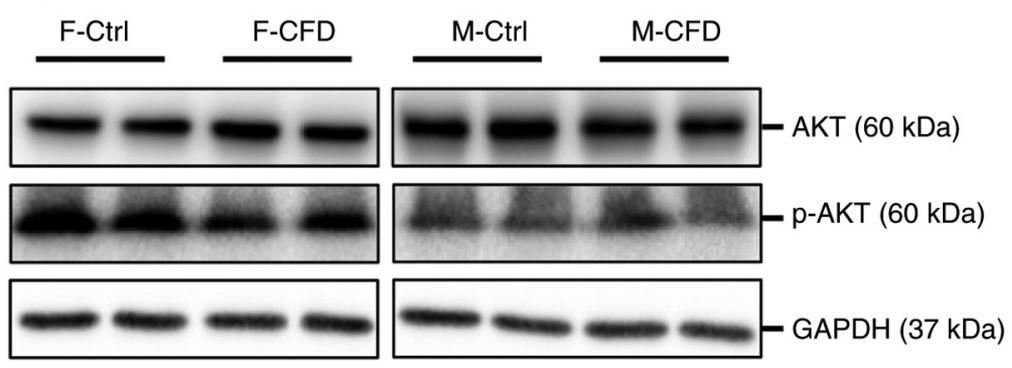

$\mathrm{D}_{\mathrm{a}}$

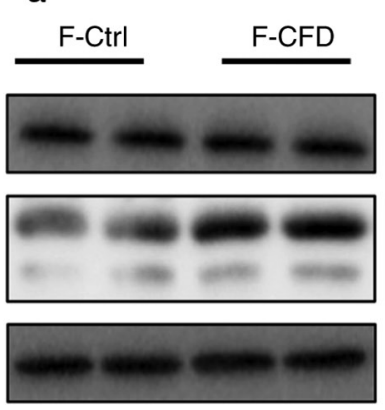

b

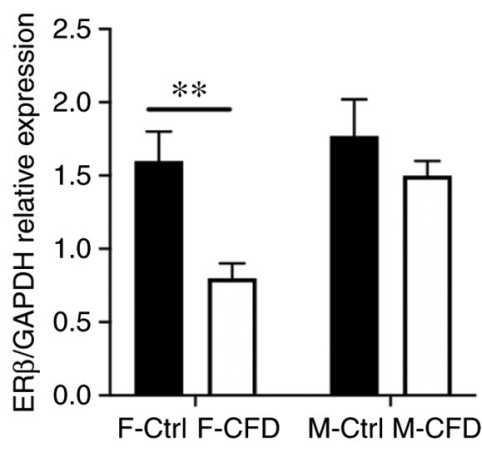

b

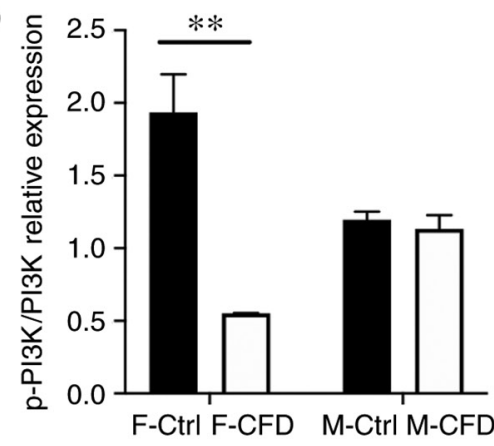

b

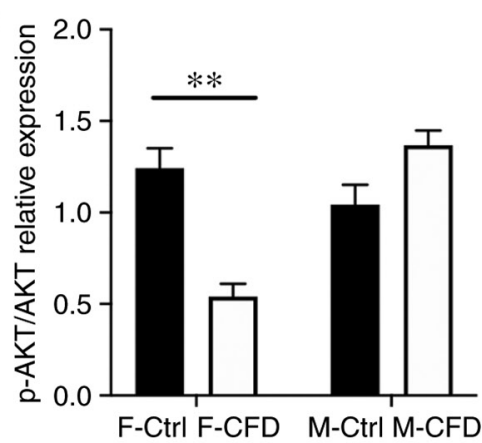

b

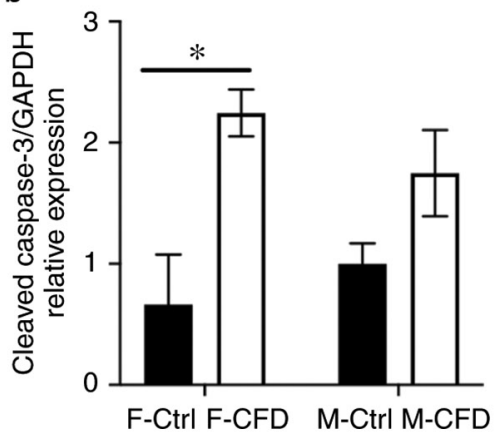

Figure 5. CFD diet inhibits PI3K/AKT signaling and upregulates cleaved caspase-3 levels in female mice. Western blot measurements of (Aa) ER $\beta$, (Ba) PI3K and p-PI3K, (Ca) AKT and p-AKT, (Da) caspase-3 and cleaved caspase-3 in brain samples. Semi-quantification of (Ab) ER $\beta,(\mathrm{Bb}) \mathrm{p}-\mathrm{PI} 3 \mathrm{~K} / \mathrm{PI} 3 \mathrm{~K},(\mathrm{Cb}) \mathrm{p}-\mathrm{AKT} /$ AKT and $(\mathrm{Db})$ cleaved caspase-3 protein expression in brain samples ( $\mathrm{n}=20$ per group). ${ }^{*} \mathrm{P}<0.05$ and ${ }^{* *} \mathrm{P}<0.01$. Data are presented as the mean $\pm \mathrm{SD}$. CFD, chronic folate-deficient; F-Ctrl, female mice with standard control diet; F-CFD, female mice with folate deficiency diet; M-Ctrl, male mice with standard control diet; M-CFD, male mice with folate deficiency diet; $\mathrm{p}$-, phosphorylated; ER $\beta$, estrogen receptor $\beta$.

but not in their male counterparts (Fig. 4C). In conclusion, folate-deficient diet caused depression-like behaviors only in F-CFD.

CFD diet elevates cleaved caspase-3 protein expression by inhibiting the PI3K/AKT signaling pathway in female mice. To investigate whether the PI3K/AKT signaling pathway was associated with cell death and the effect of E2/ER $\beta$ deficiency in mice, the protein levels of ER $\beta, p-P I 3 K, P I 3 K, p-A K T$, AKT and caspase- 3 were assessed. E2 exerts its actions via ER $\beta$ to further regulate the PI3K/AKT signaling pathway (18). The PI3K/AKT signaling pathway serves a crucial role in the regulation of cell survival, differentiation and apoptosis (21-23). Furthermore, caspase-3 is a key enzyme that is 
involved in the execution of apoptosis (24). Western blotting results revealed that the protein expression level of ER $\beta$ and the $\mathrm{p}-\mathrm{PI} 3 \mathrm{~K} / \mathrm{PI} 3 \mathrm{~K}$ expression ratio were significantly decreased in the cerebral cortex of F-CFD mice compared with those in the F-Ctrl (Fig. 5A and B). AKT is a downstream signaling molecule of PI3K $(41,42)$. However, the reduced expression ratio of $\mathrm{p}-\mathrm{PI} 3 \mathrm{~K} / \mathrm{PI} 3 \mathrm{~K}$ did not appear to affect $\mathrm{AKT}$ expression but inhibited its phosphorylation in F-CFD mice compared with that in the F-Ctrl group (Fig. 5C). Furthermore, cleaved caspase-3 (19 kDa) expression was significantly increased in F-CFD mice compared with that in the F-Ctrl group, despite total caspase-3 levels exhibiting no significant difference between F-CFD and F-Ctrl mice (Fig. 5D). No significant difference was observed in the protein expression levels of ER $\beta$, $\mathrm{p}-\mathrm{PI} 3 \mathrm{~K} / \mathrm{PI} 3 \mathrm{~K}$ and $\mathrm{p}-\mathrm{AKT} / \mathrm{AKT}$ ratios, cleaved caspase-3 or caspase-3 expression between the M-CFD and M-Ctrl groups (Fig. 5A-D). In conclusion, a folate-deficient diet reduced the expression levels of ER $\beta$ and the PI3K/AKT pathway, and increased the expression levels of cleaved caspase- 3 in the cerebral cortex only in F-CFD.

\section{Discussion}

The results of the present study revealed that CFD led to depression-like behavior in female mice but not in male mice, suggesting that CFD had a sex-dependent effect. OFT, SPT and FST were performed to explore whether CFD was involved in the depression-like behavior of mice. The results revealed that only F-CFD mice exhibited lower sucrose preferences in the SPT, longer immobility times in the FST and reduced exploratory behaviors in the OFT.

Previous studies have indicated that the differences observed in sex may be associated with E2 levels $(13,43)$. Differences in emotion processing is most apparent between men and women that are exhibiting low E2 levels. In women, lower E2 concentrations occur at certain phases of the menstrual cycle and are associated with an increasingly negative mood, increased depressive symptoms and postpartum depression $(13,43)$. Mohanty and Das (44) previously demonstrated that folate deficiency may impair the ovarian synthesis of E2 in monkeys. It has also been reported that folate deficiency can reduce circulating testosterone levels in micropigs (45). Unlike women, men generally experience consistently higher E2 activity as androgens are aromatized into E2 $(13,46)$. This process remains efficient even at low androgen concentrations (46). Although the percentage of androgen that is converted into $\mathrm{E} 2$ is $<1 \%$, the hormonal effects exhibited may remain high, since E2 is 100-1,000X more active than androgens (46). In the present study, E2 levels were measured in mouse serum using chemiluminescence. The results revealed that F-CFD mice exhibited lower E2 levels compared with those in F-Ctrl mice. Additionally, there was no significant difference in E2 levels between either groups of male mice. Hormonal differences provide the basis for proposals regarding the effects of sexual dimorphism on disorders between men and women $(3,46)$. This may explain why only F-CFD mice exhibited depression-like behavior in the present study.

Accumulating evidence has suggested that the ability of E2 to ameliorate mood symptoms is associated with ER $\beta$ activation $(18,19)$. Activated ER $\beta$ modulates the PI3K/AKT signaling pathway, which regulates both cell proliferation and cell death $(41,47)$. The present study demonstrated that decreased $E 2 / E R \beta$ expression levels in F-CFD mice led to the downregulation of the PI3K signaling pathway and upregulation of cleaved caspase-3 protein expression. Caspase-3 serves a key role in apoptosis activation (25). Liu et al (48) reported that the induction of apoptosis by tanshinone I in leukemia cells was mainly associated with the inactivation of the PI3K/AKT signaling pathway and the activation of caspase-3. Additionally, Tang et al (49) demonstrated that ursolic acid induced the apoptosis of human hepatocellular carcinoma cells by downregulating PI3K/AKT and increasing caspase-3. Zhang et al (50) also revealed that purple-colored sweet potato significantly inhibited the activity of caspase- 3 and raised $\mathrm{PI} 3 \mathrm{~K}$ and $\mathrm{p}-\mathrm{AKT}$ protein levels to exert hepatoprotective effects. The present study found that a CFD diet increased the expression levels of cleaved caspase- 3 in female mice but not male mice, suggesting that decreased E2 levels in CFD-fed female mice increased the apoptosis of cells in the cerebral cortex, which was consistent with observations previously made by Patten et al (51) and Khan et al (52). Increased cleaved caspase-3 activity promotes neuronal apoptosis $(24,52)$. A number of neural regions are involved in the processing and regulation of emotions, such as the prefrontal cortex and cingulate cortex $(53,54)$. Neuronal apoptosis decreases neurogenesis and is accompanied by the occurrence of depression-like behavior (23-25).

A recent study documented that post-weaning folate deficiency reduced the degree of maturation in neonatal hippocampal neurons and subsequently induced depression-like symptoms in male mice (55). However, the rate of hippocampal neurogenesis declines with increasing age, meaning that folate deficiency may adversely affect hippocampal function in older individuals (56). By contrast, the present study revealed that folate deficiency led to neural apoptosis in female mice through reducing the E2/ER $\beta / \mathrm{PI} 3 \mathrm{~K} / \mathrm{AKT}$ signaling pathway. This may be the underlying mechanism in which decreased neurogenesis is associated with depression-like behavior (24).

By applying animal models, the present study aimed to investigate whether folate deficiency led to differences in depression between males and females, in addition to elucidating the potential underlying mechanism by which this occurred. Unfortunately, phases of the estrous cycle were not evaluated in the behavioral assays. In addition, E2 rescue and estrogen receptor agonists are needed to examine the effects of E2 on depressive behaviors. Further study is also required to examine the effects of folate deficiency on the estrous cycle and behavior, in addition to the importance of $\mathrm{E} 2$ in this process.

In conclusion, the CFD diet led to depressive behavior only in female mice. Furthermore, the CFD diet may be associated with decreased E2 levels, which in turn inhibited the $\mathrm{PI} 3 \mathrm{~K} / \mathrm{AKT}$ signaling pathway and increased the expression levels of cleaved caspase-3, resulting in neuronal cell damage, apoptosis and depression-like behavior.

\section{Acknowledgements}

Thanks to Professor Dexiang Xu, head of the Key Laboratory of Environmental Toxicology of Anhui Higher Education 
Institutes, Anhui Medical University, China, for his technical assistance.

\section{Funding}

The present study was supported by the National Natural Science Foundation of China (grant no. 81671471), the Key Cultivation Program of School of Nursing, Anhui Medical University (grant no. hlzd2020003) and the Seedling Cultivation Program of School of Nursing, Anhui Medical University (grant no. hlqm2021006).

\section{Availability of data and materials}

The datasets used and/or analyzed during the current study are available from the corresponding author on reasonable request.

\section{Authors' contributions}

WS and QQ wrote the manuscript and performed the experiments. XC, LZ, NY and JC analyzed the data. MZ analyzed the data and revised the manuscript. WS and QQ confirm the authenticity of all the raw data. All authors read and approved the final manuscript.

\section{Ethics approval and consent to participate}

The present protocol has received ethics approval from the Ethics Committee of Anhui Medical University (approval no. LLSC20150350; Hefei, China).

\section{Patient consent for publication}

Not applicable.

\section{Competing interests}

The authors declare that they have no competing interests.

\section{References}

1. McCarron RM, Shapiro B, Rawles J and Luo J: Depression. Ann Intern Med 174: ITC65-ITC80, 2021.

2. Malhi GS and Mann JJ: Depression. Lancet 392: 2299-2312, 2018.

3. Jääskeläinen E, Juola T, Korpela H, Lehtiniemi H, Nietola M, Korkeila $\mathrm{J}$ and Miettunen J: Epidemiology of psychotic depression-systematic review and meta-analysis. Psychol Med 48: 905-918, 2018.

4. Kessler RC and Bromet EJ. The epidemiology of depression across cultures. Annu Rev Public Health 34: 119-138, 2013.

5. Bromet E, Andrade LH, Hwang I, Sampson NA, Alonso J, de Girolamo G, de Graaf R, Demyttenaere K, Hu C, Iwata N, et al: Cross-national epidemiology of DSM-IV major depressive episode. BMC Med 9: 90, 2011.

6. Reus GZ, Maciel AL, Abelaira HM, de Moura AB, de Souza TG, Dos Santos TR, Darabas AC, Parzianello M, Matos D, Abatti M, et al: $\omega-3$ and folic acid act against depressive-like behavior and oxidative damage in the brain of rats subjected to early- or late-life stress. Nutrition 53: 120-133, 2018.

7. Bender A, Hagan KE and Kingston N: The association of folate and depression: A meta-analysis. J Psychiatr Res 95: 9-18, 2017.

8. Zheng Y and Cantley LC: Toward a better understanding of folate metabolism in health and disease. J Exp Med 216: 253-266, 2019.
9. Nguyen B, Weiss P, Beydoun H and Kancherla V: Association between blood folate concentrations and depression in reproductive aged U.S. women, NHANES (2011-2012). J Affect Disord 223: 209-217, 2017.

10. Miyaki K, Song Y, Taneichi S, Tsutsumi A, Hashimoto H, Kawakami N, Takahashi M, Shimazu A, Inoue A, Kurioka S and Shimbo T: Socioeconomic status is significantly associated with the dietary intakes of folate and depression scales in Japanese workers (J-HOPE study). Nutrients 5: 565-578, 2013.

11. Huang X, Fan Y, Han X, Huang Z, Yu M, Zhang Y, Xu Q, Li X Wang X, Lu C and Xia Y: Association between serum vitamin levels and depression in U.S. adults 20 years or older based on national health and nutrition examination survey 2005-2006. Int J Environ Res Public Health 15: 1215, 2018.

12. Li Y, Gao R, Liu X, Chen X, Liao X, Geng Y, Ding Y, Wang Y and $\mathrm{He} \mathrm{J}$ : Folate deficiency could restrain decidual angiogenesis in pregnant mice. Nutrients 7: 6425-6445, 2015.

13. Albert KM and Newhouse PA: Estrogen, stress and depression: Cognitive and biological interactions. Annu Rev Clin Psychol 15: 399-423, 2019.

14. Sassarini DJ: Depression in midlife women. Maturitas 94: 149-154, 2016.

15. Li D, Li Y, Chen Y, Li H, She Y, Zhang X, Chen S, Chen W, Qiu G, Huang $\mathrm{H}$ and Zhang S: Neuroprotection of reduced thyroid hormone with increased estrogen and progestogen in postpartum depression. Biosci Rep 39: BSR20182382, 2019.

16. Xu Y, Sheng H, Bao Q, Wang Y, Lu J and Ni X: NLRP3 inflammasome activation mediates estrogen deficiency-induced depression- and anxiety-like behavior and hippocampal inflammation in mice. Brain Behav Immun 56: 175-186, 2016.

17. Liu T, Ma Y, Zhang R, Zhong H, Wang L, Zhao J, Yang L and Fan X: Resveratrol ameliorates estrogen deficiency-induced depression- and anxiety-like behaviors and hippocampal inflammation in mice. Psychopharmacology (Berl) 236: 1385-1399, 2019.

18. Gillies GE and McArthur S: Estrogen actions in the brain and the basis for differential action in men and women: A case for sex-specific medicines. Pharmacol Rev 62: 155-198, 2010.

19. Rettberg JR, Yao J and Brinton RD: Estrogen: A master regulator of bioenergetic systems in the brain and body. Front Neuroendocrino 35: 8-30, 2014.

20. Walf AA and Frye CA: A review and update of mechanisms of estrogen in the hippocampus and amygdala for anxiety and depression behavior. Neuropsychopharmacology 31: 1097-1111, 2006

21. Wang X, Yi L, Zhu Y, Zou J, Hong Y and Zheng W: AKT signaling pathway in invasive ductal carcinoma of the breast: Correlation with ERa, ER $\beta$ and HER-2 expression. Tumori 97: 185-190, 2011.

22. Hernández-Silva CD, Villegas-Pineda JC and Pereira-Suárez AL: Expression and role of the $\mathrm{G}$ protein-coupled estrogen receptor (GPR30/GPER) in the development and immune response in female reproductive cancers. Front Endocrinol (Lausanne) 11: 544, 2020.

23. Hoxhaj G and Manning BD: The PI3K-AKT network at the interface of oncogenic signalling and cancer metabolism. Nat Rev Cancer 20: 74-88, 2020.

24. Lossi L, Castagna C and Merighi A: Caspase-3 mediated cell death in the normal development of the mammalian cerebellum. Int J Mol Sci 19: 3999, 2018.

25. Sangaran PG,IbrahimZA, ChikZ, Mohamed Z and Ahmadiani A: LPS preconditioning attenuates apoptosis mechanism by inhibiting NF- $\kappa \mathrm{B}$ and caspase-3 activity: TLR4 pre-activation in the signaling pathway of LPS-induced neuroprotection. Mol Neurobiol 58: 2407-2422, 2021.

26. Phillips ML, Drevets WC, Rauch SL and Lane R: Neurobiology of emotion perception II: Implications for major psychiatric disorders. Biol Psychiatry 54: 515-528, 2003.

27. Wright AJ, Dainty JR and Finglas PM: Folic acid metabolism in human subjects revisited: Potential implications for proposed mandatory folic acid fortification in the UK. Br J Nutr 98: 667-675, 2007.

28. Reeves PG: Components of the AIN-93 diets as improvements in the AIN-76A diet. J Nutr 127(Suppl 5): S838-S841, 1997.

29. O'Leary K and Sheehy PJ: Effects of preparation and cooking of folic acid-fortified foods on the availability of folic acid in a folate depletion/repletion rat model. J Agric Food Chem 49: 4508-4512, 2001.

30. MacArthur Clark JA and Sun D: Guidelines for the ethical review of laboratory animal welfare People's Republic of China National Standard GB/T 35892-2018 (issued 6 february 2018 effective from 1 september 2018). Animal Model Exp Med 3: 103-113,2020. 
31. Bahous RH, Cosín-Tomás M, Deng L, Leclerc D, Malysheva O Ho MK, Pallàs M, Kaliman P, Bedell BJ, Caudill MA and Rozen R: Early manifestations of brain aging in mice due to low dietary folate and mild MTHFR deficiency. Mol Neurobiol 56: 4175-4191, 2019

32. Martorell AJ, Paulson AL, Suk HJ, Abdurrob F, Drummond GT, Guan W, Young JZ, Kim DN, Kritskiy O, Barker SJ, et al: Multi-sensory gamma stimulation ameliorates Alzheimer's-associated pathology and improves cognition. Cell 177: 256-271.e22, 2019.

33. Zhang M, Liu Y, Zhao M, Tang W, Wang X, Dong Z and Yu S: Depression and anxiety behaviour in a rat model of chronic migraine. J Headache Pain 18: 27, 2017.

34. Liu MY, Yin CY, Zhu LJ, Zhu XH, Xu C, Luo CX, Chen X, Zhu DY and Zhou QG: Sucrose preference test for measurement of stress-induced anhedonia in mice. Nat Protoc 13: 1686-1698, 2018.

35. Trunnell ER: Use of the forced swim test to assess 'despair'. Brain Stimul 12: 1317-1318, 2019.

36. Gao R, Ding Y, Liu X, Chen X, Wang Y, Long C, Li S, Guo L and He J: Effect of folate deficiency on promoter methylation and gene expression of Esr1, Cdh1 and Pgr, and its influence on endometrial receptivity and embryo implantation. Hum Reprod 27: 2756-2765, 2012

37. Roberts RF and Roberts WL: Performance characteristics of a recombinant enzymatic cycling assay for quantification of total homocysteine in serum or plasma. Clin Chim Acta 344: 95-99, 2004

38. Kanso $\mathrm{H}$, Inguimbert $\mathrm{N}$, Istamboulie $\mathrm{G}$, Barthelmebs $\mathrm{L}$, Calas-Blanchard C and Noguer T: Chemiluminescence immunoassays for estradiol and ethinylestradiol based on new biotinylated estrogen derivatives. Anal Biochem 537: 63-68, 2017.

39. Brandhorst G, Streit F, Kratzsch J, Schiettecatte J, Roth HJ, Luppa PB, Körner A, Kiess W, Binder L, Oellerich M and von Ahsen N: Multicenter evaluation of a new automated electrochemiluminescence immunoassay for the quantification of testosterone compared to liquid chromatography tandem mass spectrometry. Clin Biochem 44: 264-267, 2011

40. Chan YM, Bailey R and O'Connor DL: Folate. Adv Nutr 4 123-125, 2013

41. Jafari M, Ghadami E, Dadkhah $\mathrm{T}$ and Akhavan-Niaki $\mathrm{H}$ : PI3k/AKT signaling pathway: Erythropoiesis and beyond. J Cell Physiol 234: 2373-2385, 2019.

42. Fruman DA, Chiu H, Hopkins BD, Bagrodia S, Cantley LC and Abraham RT: The PI3K pathway in human disease. Cell 170: 605-635, 2017

43. Shors TJ and Leuner B: Estrogen-mediated effects on depression and memory formation in females. J Affect Disord 74: 85-96, 2003.

44. Mohanty D and Das KC: Effect of folate deficiency on the reproductive organs of female rhesus monkeys: A cytomorphological and cytokinetic study. J Nutr 112: 1565-1576, 1982.
45. Wallock-Montelius LM, Villanueva JA, Chapin RE, Conley AJ, Nguyen HP, Ames BN and Halsted CH: Chronic ethanol perturbs testicular folate metabolism and dietary folate deficiency reduces sex hormone levels in the Yucatan micropig. Biol Reprod 76: 455-465, 2007.

46. Blakemore $\mathrm{J}$ and Naftolin F: Aromatase: Contributions to physiology and disease in women and men. Physiology (Bethesda) 31: 258-269, 2016.

47. Fernandez JW, Grizzell JA and Wecker L: The role of estrogen receptor $\beta$ and nicotinic cholinergic receptors in postpartum depression. Prog Neuropsychopharmacol Biol Psychiatry 40: 199-206, 2013.

48. Liu JJ, Liu WD, Yang HZ, Zhang Y, Fang ZG, Liu PQ, Lin DJ, Xiao RZ, Hu Y, Wang CZ, et al: Inactivation of PI3k/Akt signaling pathway and activation of caspase- 3 are involved in tanshinone I-induced apoptosis in myeloid leukemia cells in vitro. Ann Hematol 89: 1089-1097, 2010.

49. Tang C, Lu YH, Xie JH, Wang F, Zou JN, Yang JS, Xing YY and Xi T: Downregulation of survivin and activation of caspase-3 through the PI3K/Akt pathway in ursolic acid-induced HepG2 cell apoptosis. Anticancer Drugs 20: 249-258, 2009.

50. Zhang ZF, Lu J, Zheng YL, Hu B, Fan SH, Wu DM, Zheng ZH, Shan Q and Liu CM: Purple sweet potato color protects mouse liver against d-galactose-induced apoptosis via inhibiting caspase-3 activation and enhancing PI3K/Akt pathway. Food Chem Toxicol 48: 2500-2507, 2010.

51. Patten RD, Pourati I, Aronovitz MJ, Baur J, Celestin F, Chen X, Michael A, Haq S, Nuedling S, Grohe C, et al: 17beta-estradiol reduces cardiomyocyte apoptosis in vivo and in vitro via activation of phospho-inositide-3 kinase/Akt signaling. Circ Re 95: 692-699, 2004.

52. Khan M, Ullah R, Rehman SU, Shah SA, Saeed K, Muhammad T, Park HY, Jo MH, Choe K, Rutten BPF and Kim MO: 17 $\beta$-estradiol modulates SIRT1 and Halts oxidative stress-mediated cognitive impairment in a male aging mouse model. Cells 8: 928, 2019.

53. Gong Q and He Y: Depression, neuroimaging and connectomics: A selective overview. Biol Psychiatry 77: 223-235, 2015.

54. Nestler EJ, Barrot M, DiLeone RJ, Eisch AJ, Gold SJ and Monteggia LM: Neurobiology of depression. Neuron 34: 13-25, 2002.

55. Nishida S, Araki R, Baba A, Asari S, Tachibana S, Nakajima Y, Iwakumo A and Yabe T: Post-weaning folate deficiency induces a depression-like state via neuronal immaturity of the dentate gyrus in mice. J Pharmacol Sci 143: 97-105, 2020.

56. Snyder JS, Soumier A, Brewer M, Pickel J and Cameron HA Adult hippocampal neurogenesis buffers stress responses and depressive behaviour. Nature 476: 458-461, 2011.

This work is licensed under a Creative Commons Attribution-NonCommercial-NoDerivatives 4.0 International (CC BY-NC-ND 4.0) License. 